\title{
Growing Kidney in the Frog
}

\author{
Techuan Chan $^{a}$ Makoto Asashima ${ }^{a, b}$

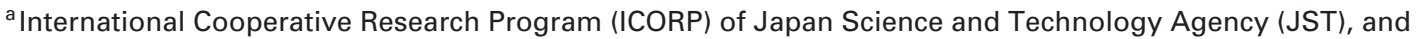 \\ ${ }^{b}$ Department of Life Sciences, Graduate School of Arts and Sciences, The University of Tokyo, Tokyo, Japan
}

\section{Key Words}

Frog, growing kidney · Pronephros development •

Kidney organogenesis · Notch signaling

\begin{abstract}
An understanding of the regulation of kidney development has increased dramatically in the past decade. The pronephros, mesonephros, and metanephros represent three distinct renal organs that function, in succession, as the vertebrate excretory system during development of the kidney. These three organ systems are derived from the intermediate mesoderm and develop in a welldefined temporal and spatial sequence. The pronephros, which consists of a tubule, duct and glomus, is established first and is the simplest of the excretory organs in vertebrates. Xenopus pronephros serves as an ideal model for investigating organogenesis and development of renal function in vertebrates. In this article, we highlight the advantages of Xenopus for analyzing kidney organogenesis and the latest research in pronephros development.
\end{abstract}

Copyright $\odot 2006$ S. Karger AG, Basel

Organogenesis is an important and attractive subject for developmental biologists. In the past decade, there has been a great deal of focus on understanding organogenesis, and many investigations of the mechanisms underlying organogenesis are reported in the literature. The kidney represents a popular and interesting model for investigating organogenesis. Vertebrates use three different kidney forms to control drainage and excrete waste throughout their lifetime: the pronephros, mesonephros and metanephros. Development of the kidney is based on successive induction of the different kidney forms. All kidneys are derived from intermediate mesoderm and develop according to an ordered temporospatial process. These unique features make the kidney an attractive model for analyzing organogenesis. In this article, we focus on Xenopus laevis, an ideal animal model for investigating vertebrate development, and review the advantages of Xenopus for analyzing kidney organogenesis, and highlight the latest research in this field.

\section{Xenopus Is a Simple and Attractive Model for Analyzing Kidney Development}

$X$. laevis, a popular animal model for developmental and cell biologists, has been studied and analyzed for many decades. Xenopus can be induced by the hormone injection to lay thousands of eggs and this can be repeated after a few months. The fertilized Xenopus eggs develop to swimming tadpoles in 4 days and commence metamorphosis within 2 months. The eggs are an ideal size to allow micromanipulation and observation of embryogenesis. In addition, a simple in vitro induction assay (fig. 1C-E) also provides an efficient and elegant system to allow investigation of the developmental process [1-3]. The presumptive ectoderm can be isolated from the late Xenopus blastula (animal cap) for the study of cell induction in an isolated environment. These features make Xenopus an excellent model for analyzing vertebrate embryogenesis.

\section{KARGER \\ Fax +4161306 1234 E-Mail karger@karger.ch} www.karger.com
(C) 2006 S. Karger AG, Basel $1660-2129 / 06 / 1033-0081 \$ 23.50 / 0$

Accessible online at: www.karger.com/nee
Dr. Makoto Asashima

International Cooperative Research Program (ICORP) of

Japan Science and Technology Agency (JST)

Komaba 3-8-1, Meguro-ku, Tokyo (Japan)

Tel. +8135454 6632, Fax +8135454 4330, asashi@bio.c.u-tokyo.ac.jp 

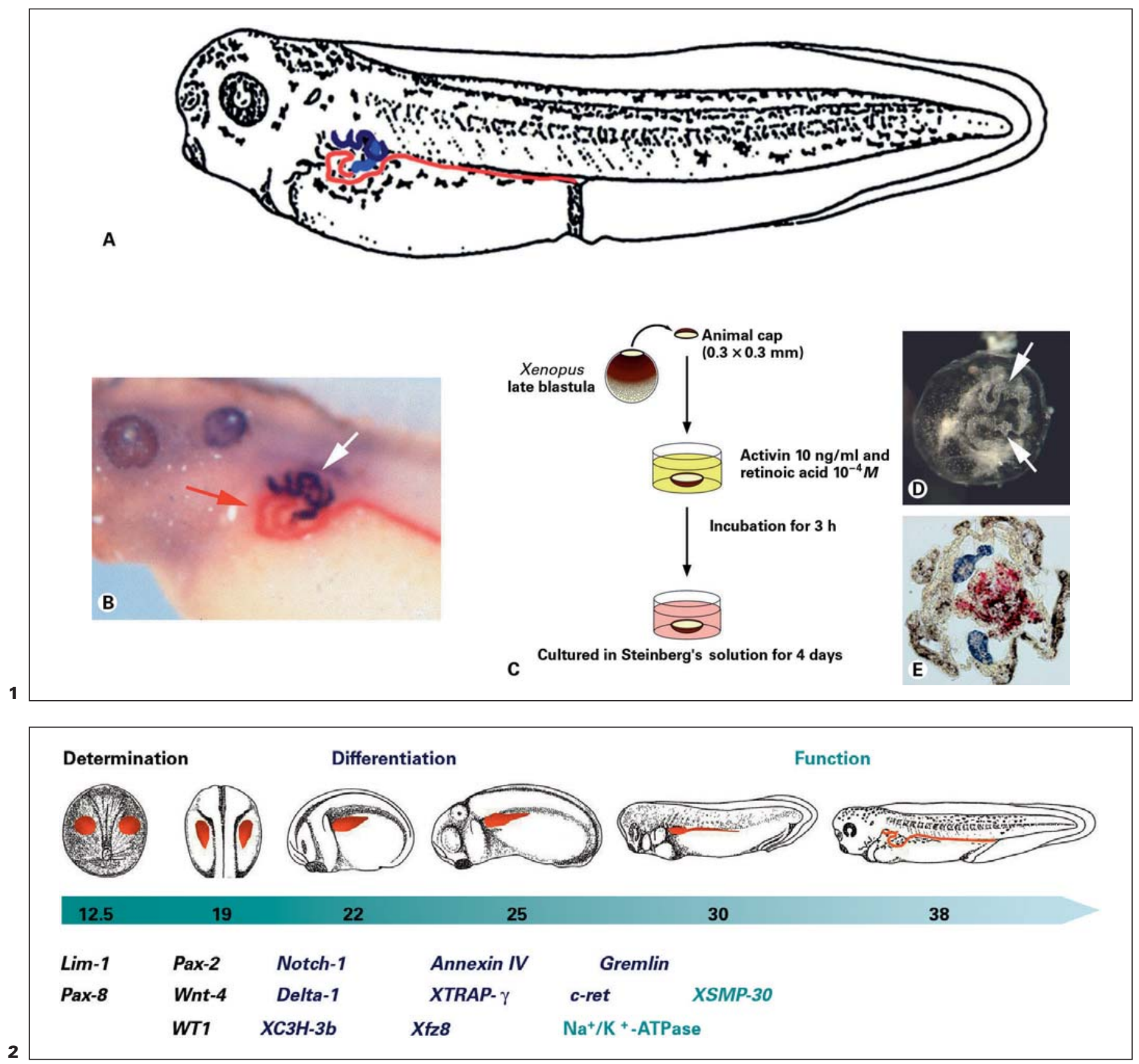

Fig. 1. Components of the pronephros in Xenopus. A The pronephros consists of a pronephric tubule (dark blue), and pronephric duct (red), the main organ of waste excretion and water balance. The glomus is not shown in this panel. B Immunostaining of the Xenopus pronephros. The pronephric tubule (white arrow) and pronephric duct (red arrow) are visualized by the pronephric tubule-specific antibody $3 \mathrm{G} 8$ and the duct-specific antibody 4A6. C-E Induction of the pronephros from activin/retinoic acid-treated Xenopus presumptive ectoderm sheets (animal caps). C Animal caps were isolated from the animal pole of Xenopus late blastulae (stage 9), immersed in activin $(10 \mathrm{ng} / \mathrm{ml})$ and retinoic acid $\left(10^{-4} M\right)$ for $3 \mathrm{~h}$, and then transferred to saline. After 4 days of culture, the pronephros was detectable in the explants by histological examination. D Ar- rows indicate the pronephric tubules in an animal cap. E Tubule (red) and duct (dark blue) in activin A/retinoic acid-treated animal cap. The pronephric tubule and duct in animal caps were detected using 3G8 and 4A6 (photograph provided by K. Osafune).

Fig. 2. Sequential gene expression in pronephros development of $X$. laevis. Colors of letters refer to the morphological stage of pronephros development. Black, dark blue, and blue refer to the determination, differentiation, and function stage in pronephros development. For example, expression of $\operatorname{Lim} 1$ and $\operatorname{Pax} 8$ commences at stage 12.5 when determination of the pronephric anlage is about to initiate, and the onset of XTRAP- $\gamma$ expression coincides with the stage the pronephric anlage undergoes differentiation. The red components in embryos represent the pronephros. 
All three vertebrate kidneys consist of a basic unit, the nephron, but differ with respect to the number and organization of the nephron within the kidney. In higher vertebrates, the pronephros and mesonephros are transient structures in embryogenesis. The nephric duct situated in the intermediate mesoderm induces a series of tubules surrounded by glomeruli in the adjacent mesoderm to form the mesonephros. Development of the metanephros is initiated by the interaction between the nephric mesenchyme and the ureteric bud on the caudal portion of the duct. Unlike the mesonephros and metanephros, development of the pronephros is not dependent on induction from the nephric duct, and the determination of tubule differentiation occurs earlier than determination of duct differentiation. Furthermore, the pronephros is the simplest and earliest form of kidney during development (fig. 1A, B). For this reason, investigations of pronephros development can provide an understanding of induction and differentiation not possible from an analysis of the metanephros.

The pronephros is the main excretory organ of Xenopus at the larval stage. The Xenopus pronephros is derived from somatic and splanchnic intermediate mesoderm and the anlage of the pronephros is formed at the late gastrula stage. Differentiation of the pronephric tubule is determined first, at the late gastrula stage, and the onset of pronephric duct differentiation proceeds from stage 14. At stage 20/21, the anlage of the pronephros becomes morphologically distinguishable. The pronephros anlage is distinctly separated from lateral mesoderm and the cells are arranged in a radical pattern from tail-bud stage. Lumenization of the tubules and anterior portion of the duct occurs at the late tail-bud stage. From stage 35/36, the entire pronephros is lumenized and starts to function. Coinciding with this stage in development is the appearance of the glomus, which can be first identified by histology on the splanchnic side of the coelom. The mesonephric cells are observed at the caudal region from stage 39. The pronephros begins to degenerate at stage 53, coinciding with initiation of mesonephros function, and has disappeared by stage 64 [4-6].

\section{Molecular Basis of Pronephros Development}

Recent reports outline the molecular basis of pronephros development (fig. 2). The activation of expression of molecules involved in pronephros development occurs successively and is accompanied by morphologic changes. $\operatorname{Lim} 1$ and $\operatorname{Pax} 8$ are the first genes expressed in the pronephros anlage and are detected from stage 12/13. Expression of $\operatorname{Lim} 1$ and Pax8 is thought to induce pronephros development. Overexpression of Lim1, Pax8, and Pax 2 leading to enlargement of the pronephros in the lateral mesoderm supports this hypothesis [7]. Another report suggests that Lim 1 is necessary for determination of tubule development and not for induction of pronephros development [8].

From the late neurula/early tail-bud stage, changes in the shape of cells in the pronephros anlage are accompanied by expression of Pax2, Wnt4, and other molecular signals. Wnts signals are known to be important for nephrogenesis. Overexpression of Wnt4 leads to fused pronephric tubules in Xenopus, and antisense morpholino oligonucleotide-mediated knockdown of $W n t 4$ results in complete loss of pronephric tubules, although the ducts are unaffected [9]. In Xenopus, morpholino-mediated knockdown of frizzled-8 $(X f z 8)$, a receptor of the Wnt protein and regulator of the activity of Wnt signals, causes a significant reduction in duct differentiation, but does not affect the expression of early pronephric maker genes in the duct region [10]. $X f_{z} 8$ is involved in both the Wnt/ $\beta$-catenin signaling pathway and the PCP pathway [1113]. It is also possible that $X f_{z} 8$ functions in duct differentiation without ligand activation. Secondary axis formation caused by overexpression of $X f z 8$ is not inhibited by Nxfz8, a secretory form of N-terminal extracellular domain of $X f_{z} 8$ [12]. Wnt-11 might be a ligand for $X f_{z} 8$ in pronephric duct development, because Wnt-11 transcripts are observed around the somites adjacent to the pronephric duct. Like $X f z 8, W n t-11$ is also reported to regulate convergent extension movement $[14,15]$. Wnt 11 is also reported to play a role in kidney development [16], although there are no reports of cooperation between Wnt- 11 and $X f z 8$.

Expression of Notch/Delta is observed in the pronephros from stage 21 [17]. Notch signaling is thought to regulate pronephric duct cell fate. Activation of Notch signaling increases the expression of tubules and glomus markers, but reduces the expression of duct markers and duct formation. Inhibition of Notch signaling also leads to an increase in the proportion of ducts formed. However, the timing of Notch expression is too late in development for it to play a role in determination of duct differentiation. Notch is expressed in the pronephros from stage 21 , and the determination of duct differentiation occurs at stage 14 . It is likely that still unknown factors are involved in regulating the fate of cells that differentiate into the tubules or ducts in development of the pronephros. 
Formation of the glomus starts at the late neurula stage. Accompanied by expression of WT1 [18], the glomus anlage separates from the tubule and duct primordium. By stage 25, molecules including annexin IV [19], $X T R A P-\gamma[20]$ and others start to be expressed. At this stage, patterning of the tubule commences and the duct extends into the cloaca. XTRAP- $\gamma$ is one of four subunits that make up the translocon-associated protein complex. The TRAP complex was thought to aid the translocation of nascent polypeptides into the lumen of the endoplasmic reticulum [21], but this was never demonstrated until now. Morpholino-mediated knockdown of XTRAP- $\gamma$ causes defects in tubulogenesis and is associated with reduced expression of the pronephros marker genes, Pax2 and $W n t 4$. The expression of $L i m 1$ is enhanced in XTRAP$\gamma$ depletion embryos. These results suggest that $X T R A P-\gamma$ regulates the translocation of molecules that in turn regulate the expression of Pax2, Wnt4, and Lim 1. XTRAP-y expression was observed in the tubules during pronephros development from stage 25 onward. As already described, the pronephros anlage is distinctly separate from the lateral mesoderm from stage 25 , and lumenization is observed in the tubular portion of the pronephros by stage 28. Coincident to these morphogenic changes, expression of $L i m 1$ is reduced and becomes localized to the tips of the pronephric tubules. These observations suggest that a reduction in the expression of Lim 1, along with maintenance of Pax2 and Wnt4 expression, is necessary for differentiation of the pronephros. It also appears that $X T R A P-\gamma$ is a regulator of pronephric tubules differentiation. At the late tail-bud stage, the anlage of the tubule is well developed and undergoes further morphogenesis. From stage 30, the expression of gremlin, dullard [22], cret [23], $\mathrm{Na}^{+} / \mathrm{K}^{+}$-ATPase [24], and other molecules, is detected in the early-lumenized pronephros.

Mesenchymal-epithelial transition is an important process in tubulogenesis during kidney development. However, the role of the mesenchymal tissue surrounding the pronephros anlage is largely unknown. A recent paper reports that factors in the mesenchymal tissue surrounding the pronephros may regulate pronephros development [25]. One of these factors is $X C 3 H-3 b$, a member of the TTP/TIS family of $\mathrm{CCCH}$ tandem zinc-finger proteins, which are physiological stimulators of instability of the mRNA encoding tumor necrosis factor- $\alpha$ in certain cell types. Expression of $\mathrm{XC} 3 \mathrm{H}-3 \mathrm{~b}$ is restricted primarily to the mesodermal tissue surrounding the pronephros. Overexpression and translational inhibition of $\mathrm{XC} 3 \mathrm{H}-3 \mathrm{~b}$ markedly and specifically inhibits pronephros development. In both cases, the expression of pronephric marker genes, such as Lim1, Pax2, Pax8, Wnt4, and WT1, is decreased and morphological development of the pronephric tubules is inhibited.

TTP/TIS zinc finger proteins can regulate stability of mRNA by binding to an AU-rich domain in the $3^{\prime}$-UTR $[26,27]$. Overexpression of $X C 3 H-3 b$ may also regulate expression of pronephros-specific genes by direct binding, although the expression of $X C 3 H-3 b$ and pronephric marker genes does not overlap and $X C 3 H$ - $3 b$ cannot bind to the mRNA of these pronephric marker genes. Moreover, the phenotype of $X C 3 H-3 b$ depletion suggests that $X C 3 H-3 b$ may regulate the expression of pronephric marker genes without regulating the stability of mRNA of the pronephric marker genes. The TTP/TIS CCCH zinc-finger protein is also reported to play a role in regulating the activity of JNK/MAPK signaling. The TTP/TIS zinc-finger protein affects apoptosis by regulating the level of TNF- $\alpha$ and the activity of JNK and MAPK/ERK [28]. These signals are known to be essential for tubulogenesis during kidney development. FGFs are also known to regulate and specify the fate of mesenchymal cells [29]. Further support for a role of $X C 3 H-3 b$ in regulating tubulogenesis via JNK/MAPK signaling comes from data that shows that deletion of $X C 3 H-3 b$ promotes phosphorylation of ERK and activity of eFGF [Chans, unpubl. data]. Chans also reports that signals from the mesenchyme surrounding the pronephros are essential for pronephros development.

\section{Conclusion}

Although many genes are expressed in the entire pronephros, including the duct, the depletion of most of these genes has no effect on pronephric duct development. Differentiation of the pronephric duct is most likely initiated by the interaction of several different molecules. It is also likely that this process involves gene redundancy such that if one of these genes is suppressed, another molecule may rescue its development. The molecular networks underlying early pronephros development have been revealed in the last 10 years, although details of the mechanisms involved are still unknown. In conclusion, Xеnоpus provides an excellent model for analyzing development of kidney and provides an efficient system to analyze the function of genes involved in this process. Corresponding analyses in higher vertebrate are much more difficult, expensive and time-consuming. The features of Xenopus provide an ideal basis for analyzing the molecular mechanisms underlying early kidney development. 


\section{References}

$\checkmark 1$ Ariizumi T, Sawamura K, Uchiyama $\mathrm{H}$ Asashima M: Dose- and time-dependent mesoderm induction and outgrowth formation by activin $\mathrm{A}$ in Xenopus laevis. Int $\mathrm{J}$ Dev Biol 1991;35:407-414.

$\checkmark 2$ Chan TC, Ariizumi T, Asashima M: A model system for organ engineering: transplantation of in vitro induced embryonic kidney. Naturwissenschaften 1999;86:224-227.

-3 Osafune K, Nishinakamura R, Komazaki S, Asashima M: In vitro induction of the pronephric duct in Xenopus explants. Dev Growth Differ 2002; 44:161-167.

4 Nieuwkoop PD, Faber J: Normal Table of Xenopus laevis (Daudin). New York, Garland, 1994.

$\checkmark 5$ Vize PD, Jones EA, Pfister R: Development of the Xenopus pronephric system. Dev Biol 1995; 171:531-540.

-6 Zhou X, Vize PD: Proximo-distal specialization of epithelial transport processes within the Xenopus pronephric kidney tubules. Dev Biol 2004;271:322-338.

7 Carroll TJ, Vize PD: Synergism between Pax-8 and lim-1 in embryonic kidney development. Dev Biol 1999;214:46-59.

$\checkmark 8$ Chan TC, Takahashi S, Asashima M: A role for Xlim-1 in pronephros development in Xenopus laevis. Dev Biol 2000;228:256-269.

$>9$ Saulnier DM, Ghanbari H, Brandli AW: Essential function of Wnt-4 for tubulogenesis in the Xenopus pronephric kidney. Dev Biol 2002;248:13-28.

$\checkmark 10$ Satow R, Chan TC, Asashima M: The role of Xenopus frizzled-8 in pronephric development. Biochem Biophys Res Commun 2004; 321:487-494.

$\checkmark 11$ Deardorff MA, Tan C, Conrad LJ, Klein PS: Frizzled-8 is expressed in the Spemann organizer and plays a role in early morphogenesis. Development 1998;125:2687-2700.

-12 Itoh K, Jacob J, Sokol YS: A role for Xenopus frizzled-8 in dorsal development. Mech Dev 1998; 74:145-157.
13 Lisovsky M, Itoh K, Sokol SY: Frizzled receptors activate a novel JNK-dependent pathway that may lead to apoptosis. Curr Biol 2002;12: 53-58.

$>14$ Heisenberg CP, Tada M, Rauch GJ, Saude L, Concha ML, Geisler R, Stemple DL, Smith JC, Wilson SW: Silberblick/Wnt11 mediates convergent extension movements during zebrafish gastrulation. Nature 2000;405:76-81.

15 Tada M, Smith JC: Xwnt11 is a target of Xenopus brachyury: regulation of gastrulation movements via Dishevelled, but not through the canonical Wnt pathway. Development 2000; 127:2227-2238.

16 Majumdar A, Vainio S, Kispert A, McMahon J, McMahon AP: Wnt11 and Ret/Gdnf pathways cooperate in regulating ureteric branching during metanephric kidney development. Development 2003;130:3175-3185.

17 McLaughlin KA, Rones MS, Mercola M: Notch regulates cell fate in the developing pronephros. Dev Biol 2000;227:567-580.

18 Wallingford JB, Carroll TJ, Vize PD: Precocious expression of the Wilms' tumor gene xWT1 inhibits embryonic kidney development in Xenopus laevis. Dev Biol 1998;202: 103-112.

19 Seville RA, Nijjarl S, Barnett MW, Masse K, Jones EA: Annexin IV (Xanx-4) has a functional role in the formation of pronephric tubules. Development 2002;129:1693-1704.

$>20$ Li DH, Chan T, Satow R, Komazaki S, Hashizume K, Asashima M: The role of XTRAP- $\gamma$ in Xenopus pronephros development. Int $\mathrm{J}$ Dev Biol 2005;49:401-408.

21 Hartmann E, Gorlich D, Kostka S, Otto A, Kraft R, Knespel S, Burger E, Rapoport TA, Prehn S: A tetrameric complex of membrane proteins in the endoplasmic reticulum. Eur J Biochem 1993;214:375-381.
22 Satow R, Chan TC, Asashima M: Molecular cloning and characterization of dullard: a novel gene required for neural development. Biochem Biophys Res Commun 2002;295:8591.

23 Durbec P, Marcos-Gutierrez CV, Kilkenny C, Grigoriou M, Wartiowaara K, Suvanto P, Smith D, Ponder B, Costantini F, Saarma M, et al: GDNF signalling through the Ret receptor tyrosine kinase. Nature 1996;381:789793.

24 Uochi T, Takahashi S, Ninomiya H, Fukui A, Asashima M: The $\mathrm{Na}^{+}, \mathrm{K}^{+}$-ATPase $\alpha$-subunit requires gastrulation in the Xenopus embryo. Dev Growth Differ 1997;39:571-580.

-25 Kaneko T, Chan T, Satow R, Fujita T, Asashima M: The isolation and characterization of $\mathrm{XC} 3 \mathrm{H}-3 \mathrm{~b}$ : a $\mathrm{CCCH}$ zinc-finger protein required for pronephros development. Biochem Biophys Res Commun 2003;308:566-572.

26 Lai WS, Carballo E, Thorn JM, Kennington $\mathrm{EA}$, Blackshear PJ: Interactions of $\mathrm{CCCH}$ zinc finger proteins with mRNA. Binding of tristetraprolin-related zinc finger proteins to Au-rich elements and destabilization of mRNA. J Biol Chem 2000;275:17827-17837.

27 Lai WS, Blackshear PJ: Interactions of CCCH zinc finger proteins with mRNA: tristetraprolin-mediated AU-rich element-dependent mRNA degradation can occur in the absence of a poly(A) tail. J Biol Chem 2001;276:2314423154.

28 Johnson BA, Blackwell TK: Multiple tristetraprolin sequence domains required to induce apoptosis and modulate responses to TNF- $\alpha$ through distinct pathways. Oncogene 2002;21: 4237-4246.

-29 Strutz F, Zeisberg M, Ziyadeh FN, Yang CQ, Kalluri R, Muller GA, Neilson EG: Role of basic fibroblast growth factor-2 in epithelial-mesenchymal transformation. Kidney Int 2002; 61:1714-1728. 